\title{
ENUCLEACIÓN PROSTÁTICA CON LÁSER HOLMIUM
}

\author{
A. Santos García-Baquero, J. Soler Martínez, F. Blanco Reina, R. Vozmediano Chicharro, P. Morales \\ Jiménez, D. Hernández Alcaraz, E. Vivas Vargas y V. Baena González.
}

Unidad Clínica de Urología. H. R. U. Carlos Haya. Málaga. España.

Resumen.- OBJETIVO: La hipertrofia prostática afecta a partir de los 40 años a un porcentaje creciente de varones, aumentando este con la edad, actualmente contamos con una nueva arma terapéutica, el láser Holmium.

MÉTODO: Recogemos de manera retrospectiva los datos obtenidos de forma retrospectiva de los 300 enfermos intervenidos mediante HoLEP prostático en nuestro centro.

RESULTADOS: Obtenemos: una estancia media hospitalaria de 1,8 días (Rango 1-15 días, mediana 1,8), un tiempo medio de cateterismo de 30,6h (Rango 12-312, Mediana 30,31 , el tiempo quirúrgico total desde que el enfermo entra hasta que sale del quirófano es de 75 min. (Rango 38- 150,
Mediana 711, El flujo máximo es de 24,7 m//seg a 6 meses y de $23,9 \mathrm{ml} / \mathrm{seg}$ a 12 meses. El rendimiento quirúrgico, los gramos resecados por minuto es de 0,48 en el grupo completo. Observamos una variación en los datos que afecta a los 20 primeros casos, con un empeoramiento de los resultados en este grupo

CONCLUSIONES: en nuestra opinión la enucleación con láser de Holmium es un método adecuado que garantiza unos resultados óptimos, perfectamente comparables a los obtenidos mediante técnicas quirúrgicas endoscópica clásicas y cirugía abierta, con un número de complicaciones bajo y que beneficia al enfermo al disminuir la necesidad de trasfusiones, el tiempo de sondaje vesical y de estancia hospitalaria, en contrapartida, presenta una curva de aprendizaje en torno a 20 procedimientos, en la que se pueden presentar complicaciones que desanimen al cirujano y que paralicen el proyecto de implantación de la técnica en un centro, al tener procedimientos fáciles y asequibles a su alcance ya establecidos.

Palabras clave: Láser Holmium. HBP. Enucleación. Láser.

Summary:- OBJECTIVES: Benign prostatic hyperplasia (BPH) affects a growing percentage of males over the age of 40 years, increasing with age. Currently, we have a new therapeutic tool available: the holmium laser.

METHODS: We retrospectively collected data of 300 patients undergoing holmium laser enucleation of the prostate ( HoLEP) in our center.

RESULTS: The results are: mean hospital stay 1,8 days /range 1-15 days, median 1,8); mean bladder catheter time 30.6 hours (range 12-312, median 30.3), total operative room time 75 minutes (range 38-150, median 71), maximal flow rate at six months $24.7 \mathrm{ml} / \mathrm{sec}$. and $23.9 \mathrm{ml} / \mathrm{sec}$. at 12 months. Surgical performance, number of grams resected per minute, 
is 0.48 for the whole group. We observed a variation in data from the first 20 cases, with worse results in this group.

CONCLUSIONS: In our opinion holmium laser enucleation is an adequate method that the guarantees optimal results, comparable to those obtained with classic endoscopic and open surgical techniques, with a low rate of complications, which benefits the patient by diminishing the need for transfusions, catheterization time, and hospital stay; conversely, it has a learning curve of around 20 procedures, which may be associated with complications that may discourage the surgeon and stop the project of technique implementation in a center, having easy, accessible, established alternative procedures.

Keywords: Holmium laser. BPH. Enucleation. Laser.

\section{INTRODUCCIÓN}

La hipertrofia benigna de próstata es una de las patologías anatomo patológicas más prevalentes en el varón (1), es edad dependiente, con aparición a partir de los 40 años (1), siendo mayor al $50 \%$ en varones mayores de 60 años y en los mayores de 85 años se encuentra próxima al $90 \%$, igualmente, los síntomas del tracto urinario inferior (STUI) asociados a la alteración histopatológica, aumentan con la edad, aproximadamente la mitad de los enfermos con los cambios histológicos presentan sintomatología moderada o severa (2).

Esta alta prevalencia de la enfermedad y de la clínica asociada ha propiciado un aumento del número de tratamientos médicos y quirúrgicos. Dentro de estos últimos, la resección transuretral de la próstata (RTUP) es el "gold standar" aunque no está exenta de morbilidad con tasas de complicaciones del $15-20 \%$ y un $10-15 \%$ requieren una segunda intervención en los 10 años siguientes (1-4). Ante esta situación hemos sido bombardeados con multitud de procedimientos, en los que se han utilizando diferentes tipos de energías (ultrasonidos, termoterapia, radiofrecuencia...).

En nuestro caso nos decidimos a emprender la aventura de la energía láser en el año 2003 y en particular del Holmium-YAG tras valorar la versatilidad del generador de energía, dentro de otra serie de procedimientos (litiasis, tumores vesicales, estenosis, etc) y por ser la única técnica validada para la intervención endoscópica de próstatas de gran tamaño frente a la prostatectomía abierta.

\section{MATERIAL Y MÉTODO}

El Láser Holmium corta y coagula con una profundidad de 0,4 mm. La difusión de energía térmica (grado de penetración) en el tejido subyacente es mínima porque la duración del pulso es de 250 microsegundos con una longitud de onda de $2140 \mathrm{~nm}$. (5.6). Con estas características el láser Holmium coagula mientras corta.

\section{Diagnóstico}

Todos los pacientes que intervenimos en nuestro servicio cumplen el protocolo diseñado por el Servicio Andaluz de Salud, Proceso HBP-Cáncer de próstata: en dicho proceso, el enfermo cuenta como mínimo con un test de sintomatología y calidad de vida (IPSS-L; Internacional Prostate Symtoms Store and Quality of live), una analítica de PSA, flujometría (salvo pacientes en retención aguda de orina) y medición de tamaño mediante ecografía transrectal. Al no tratarse este del objetivo de nuestra comunicación no nos extenderemos en los datos del proceso de diagnóstico.

\section{Protocolo}

Los enfermos acuden el día anterior para realizarse pruebas de sangre cruzada en nuestra planta de encame, vuelven a sus domicilios e ingresan el mismo día de la intervención quirúrgica a las 8:00h en ayunas, generalmente se someten a anestesia raquídea, se realiza la enucleación y salen de quirófano con sonda e irrigación continua con suero fisiológico hasta la mañana siguiente en que se retira la irrigación, si no ha ocurrido ningún problema, se retira la sonda al mediodía mañana y se espera a que orine el enfermo dos o tres veces, si no existe ningún incidente, el enfermo se da de alta por la tarde con cita para revisión en 3-4 semanas en la consulta, el mismo día de revisión, se realiza una flujometría con medición de residuo mediante bladderscam y el resultado de la anatomía patológica de la muestra obtenida durante la enucleación.

\section{Material}

- Generador de láser: versapulse powersuite (100W) con una potencia de $100 \mathrm{~W}$ (una frecuencia de $50 \mathrm{~Hz}$ y una energia de $2 \mathrm{~J}$ ).

- Cámara endoscópica.

- Resector de flujo continuo $26 \mathrm{Ch}$. con puente de adaptador de fibra láser.

- Fibra láser de 550 micras.

- Nefroscopio $26 \mathrm{Ch}$.

- Morcelador versa cut.

- Suero fisiológico para irrigación.

\section{Método quirúrgico}

Reproducimos la técnica de la enucleación prostática con láser Holmium (Holep) recogida por Gilling en 1996 (5) con algunas variaciones que nos da la experiencia acumulada. Dividimos el procedimiento en dos tipos en función de que se trate de una próstata con lóbulo medio (añadimos el $3^{\circ}$ paso) o sin él.

- Miocapsulotomía derecha desde las 7 horas desde el cuello vesical (con precaución de orificio ureteral derecho) hasta veru montagnum. En este punto practicamos incisión "en ancla" (Figura l) por detrás del veru montagnum desde las $6 \mathrm{~h}$ hasta las $9 \mathrm{~h}$.

- Miocapsulotomía izquierda (Figura 2), a las 5 horas de forma similar, desde cuello vesical a veru montagnum, con incisión "en ancla" desde las 6h hasta las $3 \mathrm{~h}$. 


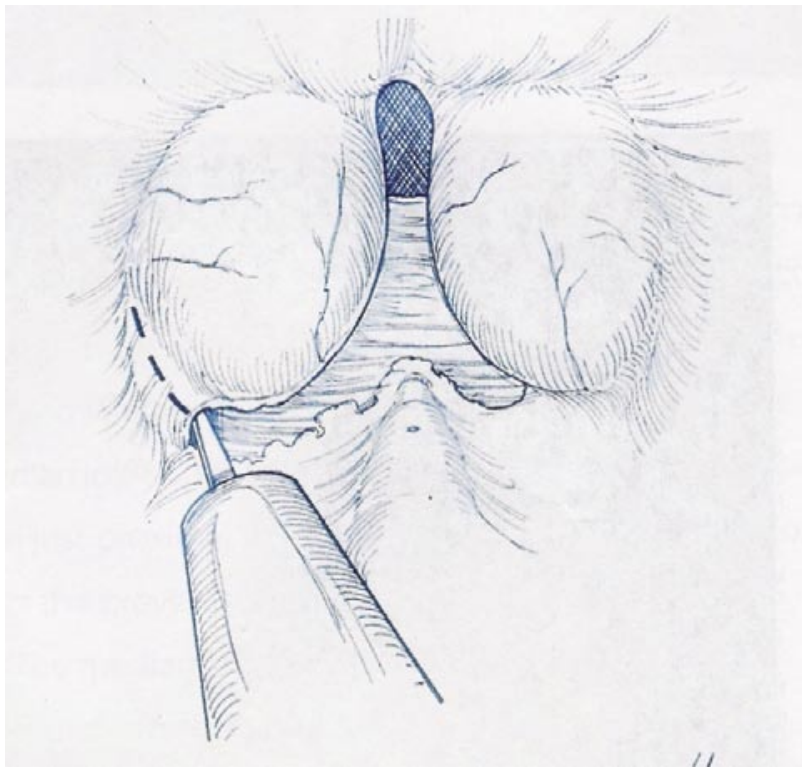

FIGURA 1. medio, intentando dirigirlo hacia arriba. Debemos de tener precaución al final de la enucleación del lóbulo medio, cuando llegamos al cuello vesical por la posibilidad de perforación y alcanzar el retrotrígono, una de las principales complicaciones en este punto de la técnica. Al final lo empujamos hasta que queda alojado en el interior de la vejiga urinaria.

- Incisión de la comisura anterior a las $12 \mathrm{~h}$ desde cuello hasta la altura del veru montagnum. Con la cual descolgamos ambos lóbulos laterales.

- Enucleación del lóbulo lateral izquierdo: debemos unir la incisión "en ancla" inferior con la superior a las $12 \mathrm{~h}$. Pensamos que es el punto más conflictivo de la intervención. De forma similar a la enucleación del lóbulo medio y de forma retrógrada enuclearemos el lóbulo lateral que introducimos en la vejiga (Figura 4).

- Enucleación del lóbulo derecho de forma similar (Figura 5).

- Vaporización y coagulación de restos prostáticos residuales y vasos sangrantes de forma exquisita (muy importante para obtener buena visión con el nefroscopio).
- Enucleación del lóbulo medio (Figura 3): os aconsejamos que previamente a la enucleación del lóbulo medio, las incisiones de las miocapsulotomias sean anchas para ir aislando el lóbulo medio y al mismo tiempo tengamos disecados la porción inferior de ambos lóbulos laterales, simplificando el procedimiento.

Una vez hecho esto, se practican incisiones transversales de un modo retrogrado, comenzando inmediatamente por detrás del veru montagnum, desde una miocapsulotomia a la otra, buscando el plano entre cápsula y adenoma. Este gesto estará facilitado si realizamos un movimiento de "delfín" del resector, por debajo del lóbulo

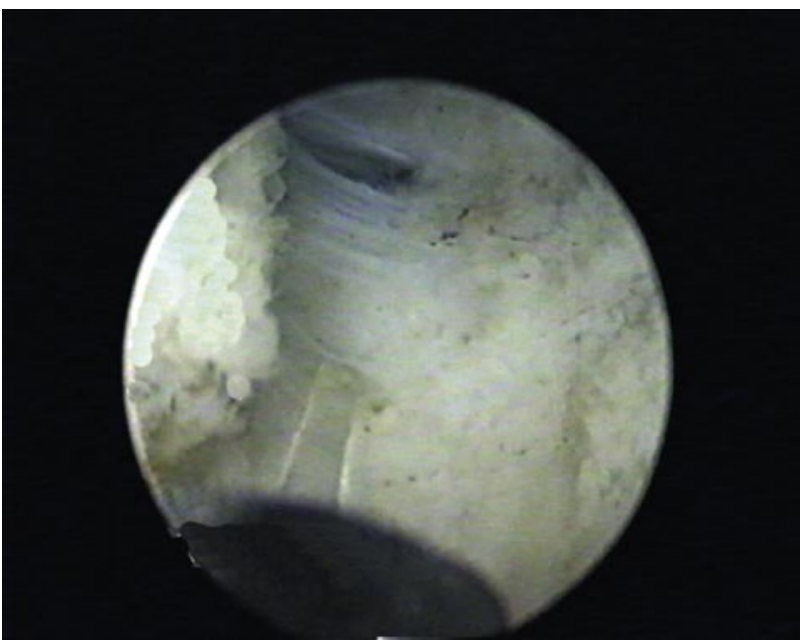

FIGURA 2

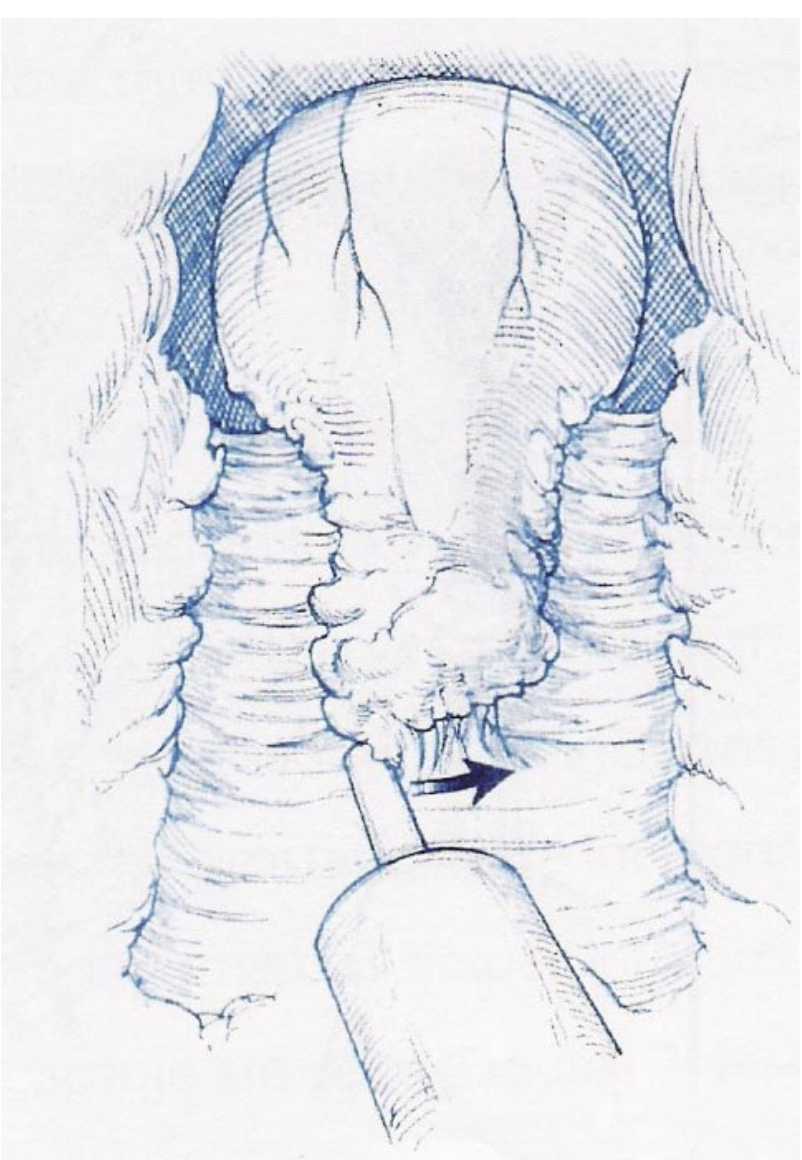

FIGURA 3. 
- Morcelación: utilizando un nefroscopio en el que se colocan dos entradas de suero salino con objeto de distender la vejiga al máximo a la vez que se aspira líquido solo por un canal de forma que protegemos la pared de lesiones yatrógenas. El pedal del morcelador presenta dos funciones: aspiración y morcelación. El primer gesto consiste en colocarse inmediatamente por detrás del cuello vesical y aspirar. Los lóbulos prostáticos contactaran con el extremo del morcelador y entonces comenzaremos la morcelación, la muestra se obtiene a partir de los fragmentos obtenidos en la morcelación.
- Posteriormente colocamos una sonda vesical del número 20-22 Ch. Con irrigación continua que retiramos en las primeras $24 \mathrm{~h}$ generalmente y alta posterior a la retirada de la misma y confirmación de que orina sin dificultad.

\section{Recogida de datos}

Realizamos un análisis retrospectivo de los datos obtenidos a partir de los pacientes intervenidos desde el inicio de la implantación de la técnica en nuestro servicio en marzo del 2003 hasta marzo del 2007 (4 años), durante este periodo hemos realizado 300 procedimientos Holep, estos pacientes presentan un seguimiento mínimo de 1 año. Se han obtenido las medias, medianas y rangos de distribución mediante el programa estadístico SPSS 11.0.

\section{RESULTADOS}

- Los datos globales (Tabla I) presentan: un peso medio de las próstatas resecado de 36 gramos (Rango 28-147 $\mathrm{cc}$, Mediana $42 \mathrm{cc}$ ) medidos por la anatomía patológica obtenida, presentaban un IPSS medio preoperatorio de 25 y un flujo medio de 7,8 $\mathrm{ml} / \mathrm{seg}$ (rango 0 (pacientes sondados)-9,6, Mediana 8,1).

- Respecto al procedimiento, obtenemos: una estancia media hospitalaria de 1,8 días (Rango 1-15 días, mediana 1,8), un tiempo medio de cateterismo de 30,6h (Rango 12-312, Mediana 30,3), el tiempo quirúrgico total desde que el enfermo entra hasta que sale del quirófano es de 75 min. (Rango 38- 150, Mediana 71), El flujo máximo es de $24,7 \mathrm{ml} / \mathrm{seg}$ a 6 meses y de $23,9 \mathrm{ml} / \mathrm{seg}$ a 12 meses. El rendimiento quirúrgico, los gramos resecados por minuto es de 0,48 en el grupo completo.

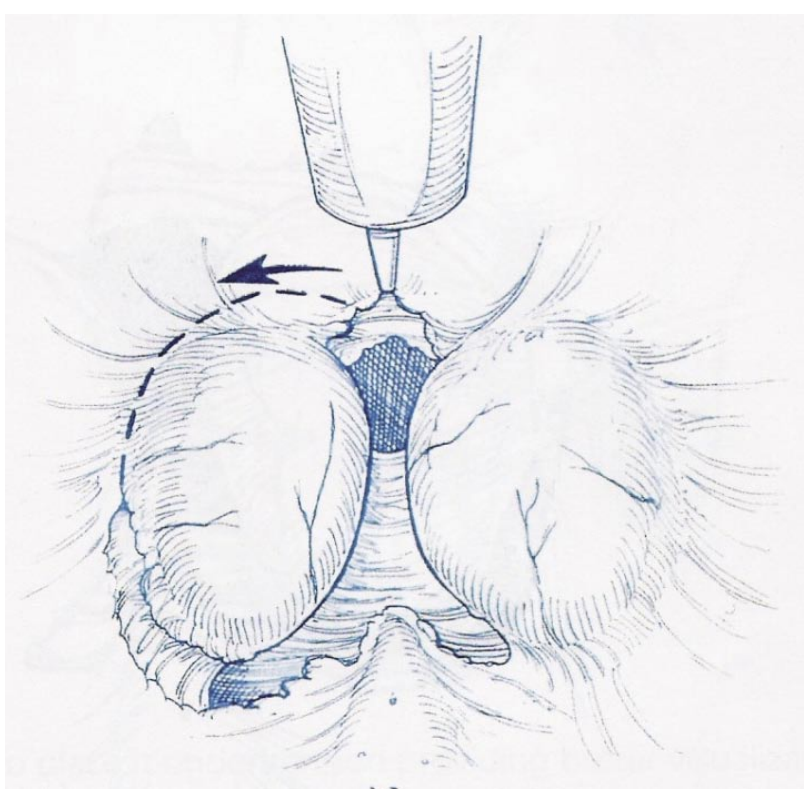

FIGURA 5. 
TABLA I. RESULTADOS GLOBALES.

\begin{tabular}{|l|l|}
\hline $\mathrm{N}$ & 300 \\
\hline Estancia Hospitalaria (días) & 1.8 \\
\hline Tiempo Cateterismo (horas) & 30.6 \\
\hline Tiempo quirúrgico (minutos) & 75 \\
\hline Q max (6 meses) & 24.7 \\
\hline IPSS (pre/post) & $25 / 4.9$ \\
\hline Peso medio (gr) & 36 \\
\hline gramos/minuto de cirugía & 0,48 \\
\hline
\end{tabular}

- Curva de aprendizaje: Analizamos los datos obtenidos distinguiendo entre los primeros 20 primeros procedimientos y el resto (Tabla II): descenso del tiempo operatorio, necesidad de transfusión, estancia media, aumento del tamaño prostático resecado, sin notar variación en el resultado final.

- Próstatas de más de 100 c.c.: Hemos realizado 60 Holep sobre próstatas con más de 100 c.c. los datos globales de estas (Tabla III) son iguales al del conjunto de datos, pero tambien hemos obtenido variación para los veinte primeros

TABLA III. PRÓSTATAS MAYORES DE 100 C.C.

\begin{tabular}{|l|l|l|l|}
\hline N & $1-60$ & $1-20$ & $21-60$ \\
\hline Tiempo operatorio (min) & 87 & 125 & 75 \\
\hline Sangrado (trasfusión) & 4 enf. & 3 enf & 1 enf. \\
\hline IPSS & 25,2 & $24 / 4$ & $26 / 5$ \\
\hline Estancia media (días) & 2,0 & 2,95 & 2,25 \\
\hline Tiempo cateterismo (horas) & 30 & 37 & 32 \\
\hline Q max a 6 meses (ml/seg) & 25,4 & 25,9 & 24,5 \\
\hline Peso medio adenoma (gr) & 110 & 107 & 112 \\
\hline gramos/minuto de cirugía & 1,26 & 0,856 & 1,49 \\
\hline
\end{tabular}

TABLA II. CURVA DE APRENDIZAJE.

\begin{tabular}{|l|l|l|}
\hline N & $1-20$ & $21-300$ \\
\hline Tiempo operatorio (min) & 105 & 80 \\
\hline Sangrado (trasfusión) & $3 \mathrm{enf}$ & 2 enf. \\
\hline IPSS & $25 / 4,9$ & $25 / 4,9$ \\
\hline Estancia media (días) & 2,85 & 1,75 \\
\hline Tiempo cateterismo (horas) & 35 & 28,7 \\
\hline Q max a 6 meses (ml/seg) & 25,9 & 25,7 \\
\hline Peso medio adenoma (gr) & 20 & 42 \\
\hline gramos/minuto de cirugía & 0,19 & 0,53 \\
\hline
\end{tabular}

procedimientos y para los restantes, con mejoría en el tiempo quirúrgico, los gramos resecados por minuto y disminución de las complicaciones y estancia media.

- Complicaciones (Tabla IV), hemos presentado: reintervenciones, sangrado medido como necesidad de transfusión sanguinea, Perforaciones de la cápsula prostática, estenosis de meato uretral y de uretra, retenciones agudas en el postoperatorio inmediato tras la retirada de la sonda vesical. Síndrome imitativo con cultivo negativo en el 1을 en el $3^{\circ}$, incontinencia urinaria.

\section{CONCLUSIONES}

En nuestra experiencia profesional durante este periodo de utilización del láser Holmium, se trata de un procedimiento que precisa una curva de aprendizaje clara, que se encuentra en torno a los 20 casos por profesional, en nuestros datos (Tabla II) se puede observar un aumento del tiempo de cirugía que ronda el $25 \%$, un aumento de la necesidad de trasfusiones en estos pacientes $3 / 20$ frente a los $2 / 280$ en el resto del grupo y un mayor tiempo de sondaje, debido a la hematuria que presentaban estos enfermos frente al resto y a la inexperiencia y dudas de los primeros procedimientos, la estancia media se ha reducido considerablemente posteriormente y los casos intervenidos no se han seleccionado tanto como los primeros, con tamaño prostáticos resecado mucho más bajos, es muy llamativo el aumento de la cantidad resecada por minuto casi $300 \%$.

Por otra parte hacemos hincapié en la diferencia entre la cirugía de próstata de tamaño $<100$ cc y las que tienen un tamaño mayor a este (Tabla III), se trata de procedimientos distintos a los previos en los que la experiencia en la cirugía de tamaños inferiores debe estar afianzada, a su vez hemos detectado un cambio importante como en 
TABLA IV. COMPLICACIONES.

\begin{tabular}{|l|c|c|}
\hline & $1-20$ & $21-300$ \\
\hline Reintervenciones & 4 & 6 \\
\hline Sangrado (transfusion) & 3 & 2 \\
\hline Perforaciones capsulares & 3 & 2 \\
\hline Estenosis meato & 2 & 5 \\
\hline Estenosis uretra & 1 & 2 \\
\hline RAO & 5 & 3 \\
\hline Sd irritativo (ler mes) & 15 & 20 \\
\hline Sd irritativo (3er mes) & 4 & 4 \\
\hline Incontinencia & 0 & 1 \\
\hline
\end{tabular}

el caso anterior a partir de aproximadamente 20 casos, la duración de la cirugía aumenta en los primeros casos frente al del grupo completo en torno al $50-60 \%$, se acumulan en este grupo la totalidad de las trasfusiones que han necesitado nuestros enfermos, aunque no había diferencias entre los grupos, los gramos resecados por minuto también son mayores que en el grupo anterior, en torno al $200 \%$.

Las complicaciones se presentan también en función de la experiencia, en la Tabla IV se puede observan el mayor acúmulo en los 20 procedimientos primeros con mayor necesidad de transfusión, mayor sintomatología irritativa, el porcentaje de pacientes en retención tras la retirada inicial de la sonda es del $25 \%$ y la necesidad de cirugía posterior del $20 \%$, todos estos datos son radicalmente distintos al del global de la serie.

\section{DISCUSIÓN}

Con respecto a las diferentes facetas que se pretenden analizar y las recomendaciones que deseamos concluir, cuando hablamos de una nueva técnica en la cirugía de la HBP, debemos analizar; las diferencias frente a lo que tenemos estandarizado: la RTU-P convencional y la cirugía abierta (adenomectomías); las complicaciones que presenta la técnica en si: el sangrado, el tiempo de cirugía; las mejoras que aporta en los parámetros que se modifican al aplicar la técnica: la estancia hospitalaria, la variación en los parámetros urodinámicos, la función sexual y por supuesto las complicaciones de la técnica.

\section{Evidencia cientifica actual}

El único metaanalisis que compara la utilización del láser, en general con la cirugía convencional, es de la fundación Cocrhane (7) iniciado en 1999. La conclusión de la misma tras comparar 20 estudios con 1989 pacientes en total es que aunque una alternativa útil no se puede dar ninguna conclusión definitiva con respecto al tipo de técnica láser idónea para el tratamiento de la HBP. En dicha revisión sólo existían 2 estudio con Láser Holmium, el realizado por Gilling en 1999 (8), que a su vez se destaca como el único láser más eficaz para la mejora del flujo urinario máximo frente a la RTU-P; y el de Kuntz del 2002 en el que se comparaba frente a cirugía abierta en próstatas con adenoma mayor de $100 \mathrm{gr}$ (9) reflejando que existe mayor disminución de la sintomatología registrada por el cuestionario de la AUA y mejores parámetros urodinámicos con HoLEP. De los 20 estudios analizados, con diferentes tipos de láser, estos dos son los únicos que aportan diferencias con respecto al resto de estudios y tipos de láser.

\section{Diferencias frente a RTU-P}

A parte de lo expuesto anteriormente encontramos varios estudios de comparación: Tan, el al. En 2003 (10) presenta su estudio randomizado comparándolo frente a RTU-P en el que concluye que existe una mejoría de los parámetros urodinámicos con HoLEP y un mayor volumen de tejido resecado con una eficiencia similar en lo que respecta a los gramos resecados por minuto de cirugía (al resecar más cantidad, precisa mayor tiempo de cirugía).

Algo que confirma también Grupta en 2006 (11) obteniendo los mismos resultados con ambas técnicas aunque empleando más tiempo con HoLEP con las mismas complicaciones. En los dos estudios se plantean las diferencias favorables en la disminución de las complicaciones sin implicaciones en la vida sexual (12)

\section{Diferencias frente a cirugía abierta}

En este apartado tenemos que volver a señalar el clásico estudio de Kuntz (9) en el que compara la adenomectomía con el HoLEP en próstatas mayores de 100 cc, concluyendo a su vez como en el caso de la RTU.P un mayor tiempo de cirugía con HoLEP pero con un volumen final similar resecado (al contrario que frente a la RTU-P) con una menor morbilidad (medido como: necesidad de transfusión sanguinea, necesidad de nuevas cirugías $u$ otro tipo de complicación) y un resultado a un año similar entre ambas técnicas con una función sexual sin cambios tras la cirugía.

Posteriormente en 2006 Naspro (13) comunica sus resultados con unas variaciones urodinamicas comparables pero con un descenso del coste del procedimiento que ronda el $10 \%$ a favor del HoLEP, aunque no explica el número medio de procedimientos medio que deben realizarse para compensar el coste del instrumental específico del láser.

\section{CONCLUSIONES}

En nuestra opinión la enucleación con láser de Holmium es un método adecuado que garantiza unos resultados óptimos, perfectamente comparables a los obteni- 
dos mediante técnicas quirúrgicas endoscópica clásicas y cirugía abierta, con un número de complicaciones bajo y que beneficia al enfermo al disminuir la necesidad de trasfusiones, el tiempo de sondaje vesical y de estancia hospitalaria, en contrapartida, presenta una curva de aprendizaje en torno a 20 procedimientos, en la que se pueden presentar complicaciones que desanimen al cirujano y que paralicen el proyecto de implantación de la técnica en un centro, al tener procedimientos fáciles y asequibles a su alcance ya establecidos.

\section{BIBLIOGRAFÍA y LECTURAS RECOMENDADAS ("lectura de interés $\mathrm{y}^{* *}$ lectura fundamental)}

1. KIRBY, R.S.: "The natural history of binign prostatic hyperplasia: What have learned in the last decade?". Urology, 56: 3, 2000.

2. DUNSMUIR,W.D.; EMBERTON, M.; THE NATIONAL PROSTAECTOMY AUDIT STEERING GROUP.: "There is significant sexual dysfuntion following TURP”. Br. J. Urol., 77: 39, 1996.

3. MEBUST, W.K.; HOLTGREWE, H.L.; COCKETT, A.T.K. y cols.: "Transuretral prostatectomy: Inmediate and postoperative complications. A cooperative study of 13 participating institutions avaluating 3.885 patients". J. Urol., 141: 243, 1989.

4. HOLTGREWE, H.L.; VALK, W.L.: "Factors influencing the mortality and morbidity of transurethral prostatectomy: A study of 2.1015 cases". J. Urol., 87: 450, 1962.

5. CASSC, B.; CRESSWELL,W.D.; FRAUNDORFER, M.R.: "Holmium laser resection of prostate: Preliminary results of a new method for treatment of benign prostatichyperplasia". Urology, 47: 48, 1996.
6. ERHARD, M.J.; BAGLEY, D.H.: "Urologic applications of the holmium laser: Preliminary experience". J. Endourol., 9: 383, 1995.

7. HOFFMAN, R.M.; MAcDONALD, R.; KILT, T.J.: "Prostatectomía con láser para la obstrucción prostática Benigna. (Revisión Cochrane traducida)". La biblioteca Cochrane Plus, 2008 Numero 1. Oxford: Update Software Ltd. Disponible en: http://WWW.update-software. com. (traducida de The Cochrane Library, 2008 Issue 1. Chichester, UK: John Wiley \& Sons, LTD.) 2008.

**8. GILLING, P.J.; MacKEY, M.; CRESSWEL, M. y cols.: "Holmium laser versus transurethral resection of the prostate: A randomized prospective trial with 1-year follow-up". J. Urol., 162: 1640, 1999.

**9. KUNTZ, R.M.; LEHRICH, K.: "Transuretral Holmium laser enucleation versus transvesical open enucleation for prostate adenoma greater then $100 \mathrm{gm}$ : A randomized prospective trial of 120 patients". J. Urol., 168: 1465, 2002.

*10. TAN, A.H.; GILLING, P.J.; KENNETT, K.M. y cols.: "A randomized trial comparing holmium laser enucleation of the prostate with transurethral resection of the prostate for the treatment of bladder outlet obstruction secondary to benign prostatic hyperplasia in large glands (40 to 200 grams)". J. Urol., 170: 1270, 2003.

11. GUPTA, N.; SIVARAMA, K.; KUMAR, R. y cols.: "Comparison of standard transurethral resection, transurethral vapour resection and holmium laser enucleaction of the prostate for managing benign prostatic hyperplasia of $>40$ g”. BJU Int., 97: 85, 2006.

12. BRIGANTI, A.; NASPRO, R.; GALLINA, A. y cols.: "Impact on sexual function of holmium laser enucleation versus transurethral resection of the prostate: Results of a prospective, 2-center, randomized trial". J. Urol., 175: 1817, 2006.

*13. NASPRO, R.; SUARDI, N.; SALONIA, A. y cols.: "Holmium Laser enucleation of the prostate versus open prostatectomy for prostates $>70 \mathrm{~g}: 24$ month follow-up”. Eur. Urol., 50: 563, 2006. 